\title{
¿POR QUÉ IMPORTA LA FILOSOFÍA HOY?
}

A continuación ofrecemos al lector la transcripción del Seminario "Por qué importa la filosofía hoy", que se llevó a cabo en la Facultad de Filosofía y Humanidades de la Universidad de Chile el 4 de diciembre de 2018. Este encuentro se desarrolló en torno a dos paneles. El primero, ¿por qué importa la filosofía en la universidad y en la cultura de nuestro tiempo?, fue encabezado por el profesor Carlos Peña González (Universidad Diego Portales), autor del libro Por qué importa la filosofía (Taurus 2018). Participaron en la discusión los profesores Jorge Acevedo Guerra (Universidad de Chile), Diana Aurenque Stephan (Universidad de Santiago), Gustavo Cataldo Sanguinetti (Universidad Andrés Bello) y Jaime Araos San Martín (Universidad de Chile).

El segundo, ¿por qué importa la filosofía en la educación escolar del siglo XXI?, fue encabezado por la investigadora Sylvia Eyzaguirre Tafra (Centro de Estudios Públicos), autora, entre otros estudios sobre educación, del artículo "El rol de la filosofía en el desarrollo del pensamiento crítico" (Puntos de Referencia $\mathrm{N}^{\circ}$ 485, CEP julio 2018, 1-8). En este panel participaron las profesoras Marcela Gaete Vergara (Universidad de Chile) y Marta Almeida U. (Santiago College), así como el profesor Augusto Astudillo Cánobra (REPROFICH, Colegio Extremadura, IP Santo Tomás y Penta UC).

Queremos agradecer el invaluable apoyo que dio en todo momento a la realización de este seminario el Decano de la Facultad de Filosofía y Humanidades, profesor Carlos Ruiz Schneider. Agradecemos también a la Revista de Filosofía, dirigida por el profesor Marcos García de la Huerta, el interés y ayuda que nos dio para publicar los textos respectivos en tan prestigioso espacio de investigación y discusión filosófica. Y queremos agradecer a los panelistas, a los asistentes, a la dirección de Extensión de la Facultad y a todos quienes colaboraron de uno u otro modo para que el Seminario haya logrado el éxito que muy notoriamente alcanzó.

Jaime Araos S. M.

Organizador 